\title{
A narrative review of multiple endocrine neoplasia syndromes: genetics, clinical features, imaging findings, and diagnosis
}

\author{
Xuefang Hu ${ }^{1 \#}$, Jian Guan ${ }^{1 \#}$, Yangdi Wang ${ }^{1}$, Siya Shi ${ }^{1}$, Chenyu Song ${ }^{1}$, Zi-Ping Li ${ }^{1}$, Shi-Ting Feng ${ }^{1}$, \\ Jie Chen ${ }^{2}$, Yanji Luo ${ }^{1 \wedge}$ \\ ${ }^{1}$ Department of Radiology, The First Affiliated Hospital of Sun Yat-Sen University, Guangzhou, Guangdong, China; ${ }^{2}$ Department of \\ Gastroenterology, The First Affiliated Hospital of Sun Yat-Sen University, Guangzhou, Guangdong, China \\ Contributions: (I) Conception and design: X Hu, J Guan, J Chen, Y Luo; (II) Administrative support: ZP Li, ST Feng; (III) Provision of study \\ materials or patients: Y Wang, C Song, S Shi; (IV) Collection and assembly of data: X Hu, J Guan; (V) Data analysis and interpretation: X Hu, J \\ Guan, J Chen, Y Luo; (VI) Manuscript writing: All authors; (VII) Final approval of manuscript: All authors. \\ \#These authors contributed equally to this work. \\ Correspondence to: Jie Chen, MD, PhD. Department of Gastroenterology, The First Affiliated Hospital, Sun Yat-Sen University, 58 Zhongshan Road \\ 2, Guangzhou 510080, China. Email: chenjie7@mail.sysu.edu.cn; Yanji Luo, MD, PhD. Department of Radiology, The First Affiliated Hospital, Sun \\ Yat-Sen University, 58 Zhongshan Road 2, Guangzhou 510080, China. Email: luoyj26@mail.sysu.edu.cn.
}

Objective: We aimed to provide ideas for clinicians, especially radiologists, for the diagnosis of multiple endocrine neoplasia (MEN) syndromes.

Background: MEN syndromes include MEN1, MEN2, and MEN4 and usually involve 2 or more endocrine tumors. The MEN syndromes are a group of euchromatic dominant genetic diseases, and the main genes involved include MEN1 (MEN1), RET (MEN2), and CDKN1B (MEN4).

Methods: In this article, involving 8 cases ( 4 cases of MEN1, 2 cases of MEN2A, 1 case of MEN2B, 1 case of MEN4) from our center, we introduced the disease spectrum, clinical manifestations (especially imaging findings), and related genes involved in each type of MEN syndromes. We also discussed the differential diagnosis between MEN and sporadic tumors and emphasized that MEN should be screened and the relevant required examinations.

Conclusions: Considering that MEN syndromes involve multiple endocrine gland tumors and nonendocrine organ diseases, it is very important to identify potential patients early and perform multiple examinations on them, including biochemical and multitype, and multisite imaging examinations according to the disease spectrum of each type. Considering that this is a group of genetic diseases, both interviewing patients about their family history and genetic testing are also very important. Only in this way can a comprehensive and accurate diagnosis be made, enabling patients to receive appropriate treatment and improve their prognosis.

Keywords: Multiple endocrine neoplasias (MENs); endocrine glands; computed tomography (CT); magnetic resonance imaging (MRI)

Submitted Mar 12, 2021. Accepted for publication Apr 29, 2021.

doi: $10.21037 /$ atm-21-1165

View this article at: http://dx.doi.org/10.21037/atm-21-1165

$\wedge$ ORCID: 0000-0002-8514-020X. 


\section{Introduction}

As a group of heterogeneous clinical syndromes, multiple endocrine neoplasia (MEN) syndromes are characterized by the occurrence of 2 or more endocrine gland tumors in a patient, either simultaneously or successively. The MEN syndromes are a group of autosomal dominant genetic disorders with high penetrance. Cases usually present within familial clusters, but sporadic cases do exist. The MEN syndromes include MEN1, MEN2, and MEN4: MEN1 is related to the inactivating mutation of the tumor suppressor gene MEN1 on chromosome 11 (1); MEN2 is related to the activating mutation of the rearranged during transfection (RET) proto-oncogene on chromosome 10 (2); MEN4 is related to the inactivating mutation of the cyclindependent kinase (CDK) inhibitor $1 \mathrm{~b}(C D K N 1 B)$ gene on chromosome 12 (3). It is worth noting that MEN2 is divided into 3 subtypes according to different phenotypes, including MEN2A, MEN2B, and family medullary thyroid carcinoma (FMTC). The tumors involved in MEN1 are predominantly parathyroid tumors, gastro-enteropancreatic neuroendocrine tumors, and pituitary tumors. Patients with MEN2A often develop medullary thyroid carcinoma (MTC) and pheochromocytoma. MEN2B also mainly involves MTC and pheochromocytoma. Some special clinical manifestations can be found in patients with MEN2B (such as "Marfanoid" body habitus, multiple mucosal neuromas on lips and tongue) (4). The FMTC generally exclusively involves MTC. The clinical manifestations of MEN4 are similar to those of MEN1, but the related mutant genes are different.

Involving multiple endocrine glands and nonendocrine organ lesions, the clinical manifestations of MEN are complex and diverse. Due to the diversity of its manifestations, it is not easy to diagnose MEN at the first visit clinically. Clinicians must identify the relevant clinical manifestations of patients with MENs as early as possible and perform various subsequent examinations, including related biochemical examinations and multitype and multisite imaging. Considering that this is a group of genetic diseases, both interviewing patients about their family history and genetic testing are very important. Accurate and comprehensive diagnosis will enable patients to receive appropriate treatment and clinical management. Grajo et al. introduced the imaging manifestations of various organ lesions involved in MEN (5). McDonnell et al. briefly described the clinical manifestations of MEN and emphasized when MEN should be screened (6). In this article, involving 8 cases ( 4 cases of MEN1, 2 cases of MEN2A, 1 case of MEN2B, 1 case of MEN4) from our center, we introduced the disease spectrum, clinical manifestations (including symptoms/signs; laboratory examinations; various imaging manifestations) and related genes involved in each type of MEN. We also discussed the differential diagnosis between MEN and sporadic tumors and emphasized that MEN should be screened and the relevant required examinations. This article can provide ideas for clinicians to diagnose MEN. We present the following article in accordance with the Narrative Review reporting checklist (available at http://dx.doi.org/10.21037/ atm-21-1165).

\section{Genetics}

\section{MEN1 gene}

The tumor suppressor gene MEN1, located on chromosome $11 \mathrm{q} 13$, has 10 exons and encodes the menin protein, regulating transcription, cell division, and proliferation. The pathogenesis of MEN1 is Knudson's 2-hit hypothesis $(6,7)$. This means that when born with an abnormal allele, the patient does not develop the disease due to the existence of the other normal allele, and the disease occurs when the other allele is mutated at the somatic level. The mutation sites of the MEN1 gene are located in and around the open reading frame, and more than 1,300 mutation sites have been discovered (8). Although MEN1 mutations are mostly hereditary, 10\% of MEN1 cases have a de novo germline (sporadic cases without a family history) (9). In $20-30 \%$ of MEN1 patients, germline mutations were not detected in the coding region of $M E N 1$, which may be caused by gene deletions, noncoding region mutations, or other gene mutations (9). To date, an obvious genotype-phenotype correlation has not been found in MEN1, which indicates no correlation between the mutation site and the phenotype (7). A study has shown that MEN1 promoter methylation may be associated with the severity of disease (10). Genetic testing is very important for the early diagnosis and identification of asymptomatic carriers (11).

\section{RET gene}

The proto-oncogene RET, with 21 exons, is located on chromosome 10q11.2 and encodes a membrane-bound tyrosine kinase cell surface receptor (6). Mutations of the RET gene are present in $98 \%$ of MEN2 patients (7). The 
de novo germline is also present in MEN2 patients, including MEN2A (15\%), MEN2B (50\%), and FMTC (10\%) (12). Significant genotype-phenotype correlations have been detected in MEN2 patients $(7,9)$. Mutations in exon 10 and 11 are those that are usually associated with MEN2A. Mutations in exon 16 can usually be found in patients with MEN2B. The occurrence of FMTC is often related to exons 10, 13, and 14 (12). Hirschsprung disease can occur in some patients with MEN2, usually related to exon 10 mutations. A tenth of MEN2A families has cutaneous lichen amyloidosis, which is often related to exon 11 (13). Genetic testing of RET can confirm the diagnosis and predict the potential phenotype, which can guide clinical management $(14,15)$. Hierarchical gene management of MEN2-related MTC can greatly facilitate the improvement of prognosis. According to the RET mutation site, the American Thyroid Association (ATA) divided the disease risk into 4 levels. From level A to level D, the phenotypic severity gradually increased. The codons involved are as follows: A: 768, 790, 791, 804, 649, 891; B: 609, 611, 618, 620, 630, 631; C: 634; D: 918, 883. Patients with level A risk are recommended to undergo total thyroidectomy after age 5 or when computed tomography (CT) results are positive. Patients with level $\mathrm{B}$ or $\mathrm{C}$ risk should receive total thyroidectomy before age 5. Patients with level D risk should have surgery as early as their first year of life (9).

\section{CDKN1B gene}

The tumor suppressor gene $C D K N 1 B$, located at $12 \mathrm{q} 13$, encodes nuclear protein p27, which is a CDK inhibitor that can regulate the transition of cells from $\mathrm{G} 1$ phase to $\mathrm{S}$ phase (3). The phenotypes of MEN4 and MEN1 are similar. Approximately $3 \%$ of patients clinically diagnosed with MEN1 do not have MEN1 mutations but carry $C D K N 1 B$ mutations. There have been few cases reported in China and internationally, and 17 mutations have been found. Due to the small number of cases, the genotype-phenotype relationship cannot be assessed $(16,17)$.

\section{Clinical features and imaging findings}

\section{MEN1}

The MEN1 syndrome is also called Wermer syndrome, with an estimated prevalence of 2-3 per 100,000 (9). The predominant tumors involved in MEN1 include parathyroid, gastro-entero-pancreatic endocrine, and pituitary tumors (Figures 1-4). Patients with MEN1 also often develop cutaneous tumors, such as lipomas, collagen tumors, and fibrovascular facial tumors. The detection of skin lesions is helpful for early diagnosis. Other tumor types are associated with MEN1, including adrenal cortex tumors, foregut carcinoids, thyroid tumors, and meningiomas. In recent years, ovarian and uterine tumors related to MEN1 have also been reported (18-20). Studies have found that women with MEN1 have a significantly higher risk of breast cancer (21). Neuroendocrine tumors of the pancreas, thymus, and bronchi are the main causes of death in MEN1 patients (22).

\section{Parathyroid tumors}

Hyperparathyroidism (HPT) occurs in 90\% of MEN1 patients, and it is the most common and earliest manifestation of MEN1 (7). Most of its pathological types are adenomas or hyperplasia, which often involve 2 or more parathyroid glands. The typical age of parathyroid adenomas is 20-25 years old, which is significantly younger than that of non-MEN1-related parathyroid adenomas (7). The presence of HPT can be clinically asymptomatic and only manifest as biochemical abnormalities. It can also manifest as hypercalcemia (drowsiness, fatigue, depression, constipation, polydipsia, polyuria), urinary tract stones, osteoporosis (pathological fractures), and occasionally peptic ulcers.

In laboratory examinations, an increase of serum calcium, a decrease of serum phosphorus, and an increase of parathyroid hormone concentration often indicate HPT. In terms of clinical imaging, neck ultrasound and 99mTc-Sestamibi scan are often used (1). In ultrasonography, a uniform low echo with an oval shape (large adenomas can be lobular) relative to the thyroid gland can be observed, and marginal blood vessels can sometimes be observed. In 99m Tc-Sestamibi scan delayed imaging, the tracer is abnormally aggregated at the lesion (Figures 1A,2A,4A) (5). A diagnosis of MEN1 diagnosis should be considered when pathological fractures occur in young patients with endocrine disorders (23).

\section{Gastro-entero-pancreatic endocrine tumors}

Gastro-entero-pancreatic endocrine tumors occur in 30-80\% of patients with MEN1 and are mostly multifocal, usually on the pancreas and duodenum (7). Among gastro-enteropancreatic endocrine tumors, gastrinomas are the most common, followed by nonfunctional tumors and insulinomas. There are also some rare tumor types, such as glucagon tumors and vasoactive intestinal peptide tumors (6).

Gastrinomas are mostly malignant, and half of such 

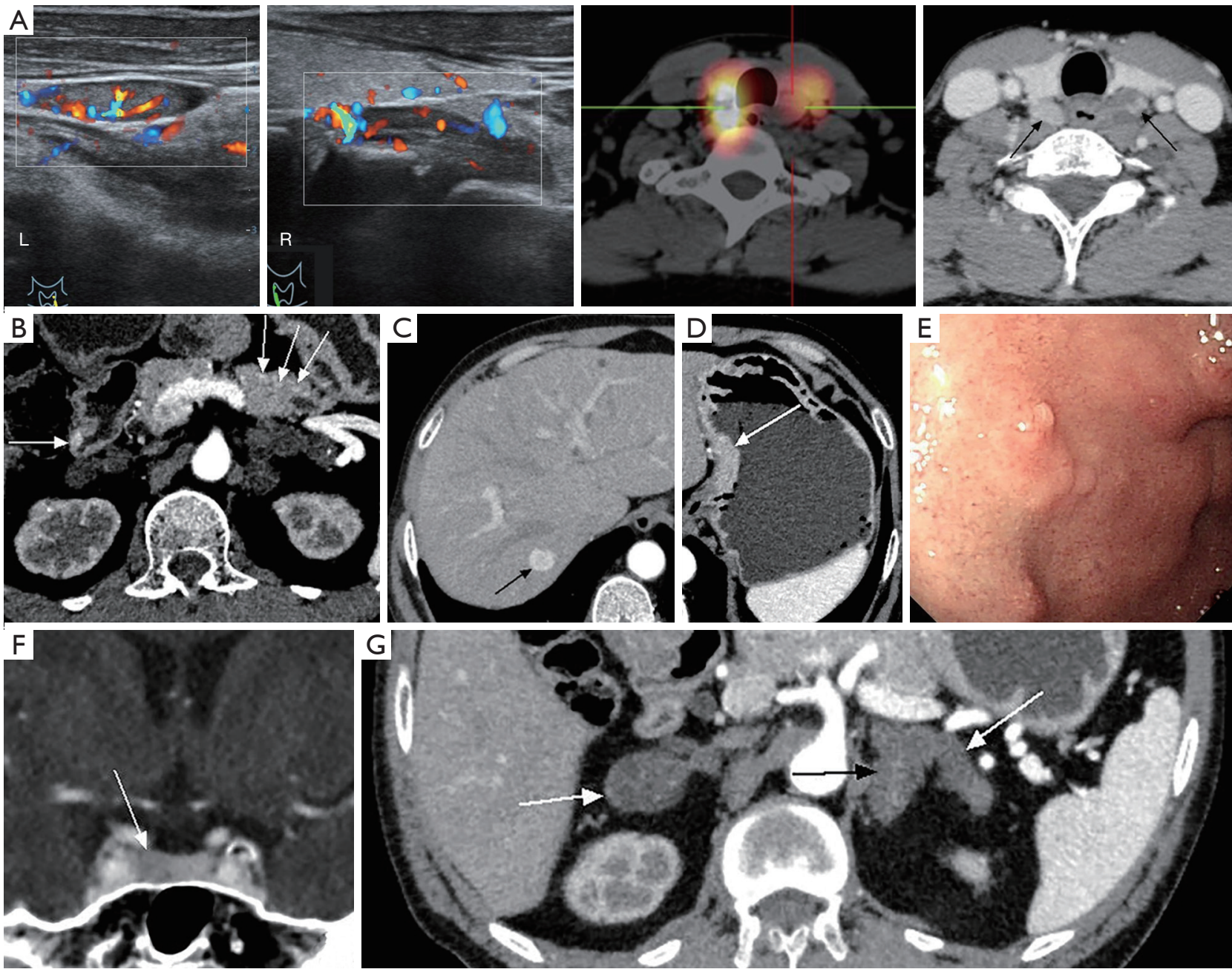

Figure 1 A 49-year-old male patient presented with recurrent diarrhea and was later diagnosed with MEN1. (A) Ultrasound image showed that there were hypoechoic nodules with rich blood flow in bilateral parathyroid areas. 99mTc-MIBI fusion images showed high uptake nodules in bilateral parathyroid areas. An axial contrast-enhanced CT image showed mildly enhanced nodules (arrows) in the posteriorinferior part of the thyroid gland. The nodules were pathologically confirmed as parathyroid adenomas. (B) Axial contrast-enhanced CT image showed multiple small enhanced nodules (arrows) in the gastroduodenal wall and tail of the pancreas, which was pathologically proved to be neuroendocrine tumors (gastrinoma). (C) Axial contrast-enhanced CT image showed a significantly enhanced nodule (arrow) in the S7 segment of the liver, which was pathologically proved to be hepatic metastasis of gastrinoma. (D) Axial contrast-enhanced CT image showed mucosal hypertrophy of the lesser curvature of the gastric body (arrow). (E) Gastroscopy showed multiple small polypoid protuberances on the gastric fundus mucosa, which was pathologically confirmed as gastric neuroendocrine neoplasms type II. (F) On the contrast-enhanced coronal reconstruction image, an enhanced nodule (arrow) was seen on the right side of the pituitary, which was less enhanced than the surrounding normal tissue. The lesion was pathologically confirmed as pituitary microadenoma. (G) Axial contrast-enhanced image showed nodular thickening of bilateral adrenal glands (arrows), which was pathologically confirmed as nonfunctional adenoma. The patient's genetic sequencing revealed a heterozygous pathogenic mutation in MEN1. MEN1, multiple endocrine neoplasia type 1; CT, computed tomography.

patients have already metastasized at first diagnosis. The age of onset was shown to be 20 years earlier than that of sporadic cases $(6,7)$. The clinical manifestation of gastrinoma is Zollinger-Ellison syndrome, characterized by hypergastrinemia, excessive gastric acid production, peptic ulcers, and diarrhea. Peptic ulcers are always multiple 

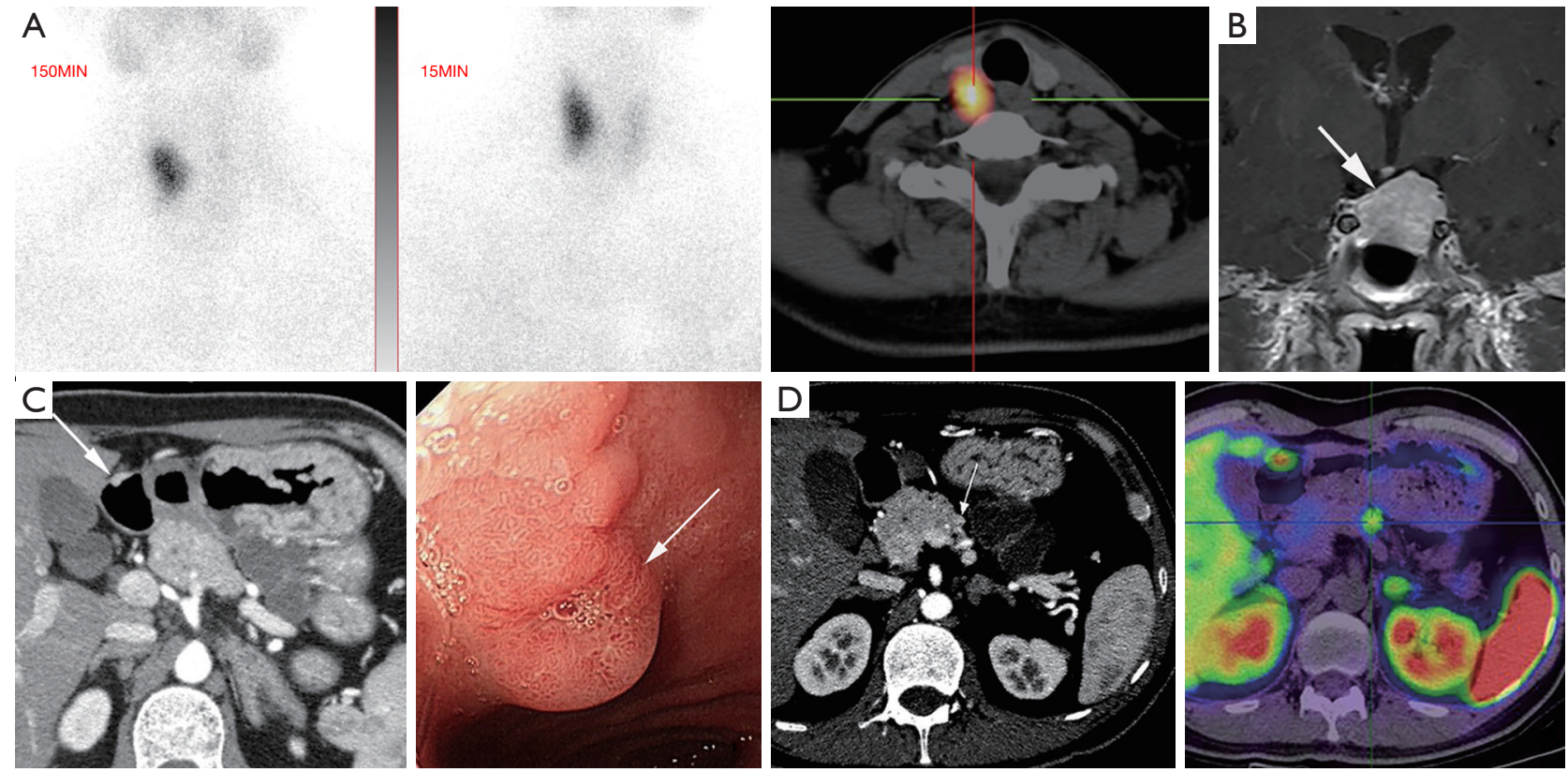

Figure 2 A 37-year-old male patient presented with acid reflux and vomiting and was later diagnosed with MEN1. (A) 99mTc-MIBI image of the right parathyroid gland was positive, which was pathologically confirmed as parathyroid adenoma. (B) Coronal contrastenhanced $T_{1}$ WI image showed a circular mass (arrow) in the left sellar with uneven and obvious enhancement. The lesion was pathologically confirmed as pituitary macroadenoma. (C) Axial contrast-enhanced CT image showed an enhanced nodule (arrow) in the duodenal bulb and the thickened left adrenal gland. Gastroscopy image showed a mucosal bulge (arrow) approximately $6 \mathrm{~mm} \times 5 \mathrm{~mm}$ on the duodenal bulb. The lesion was pathologically shown to be a gastrinoma. (D) Axial contrast-enhanced CT image showed an enhanced nodule (arrow) in the neck of the pancreas. The somatostatin receptor scintigraphy was positive in the corresponding region. The lesion was pathologically shown to be a gastrinoma. MEN1, multiple endocrine neoplasia type 1; CT, computed tomography; $\mathrm{T}_{1} \mathrm{WI}, \mathrm{T}_{1}$ weighted imaging.
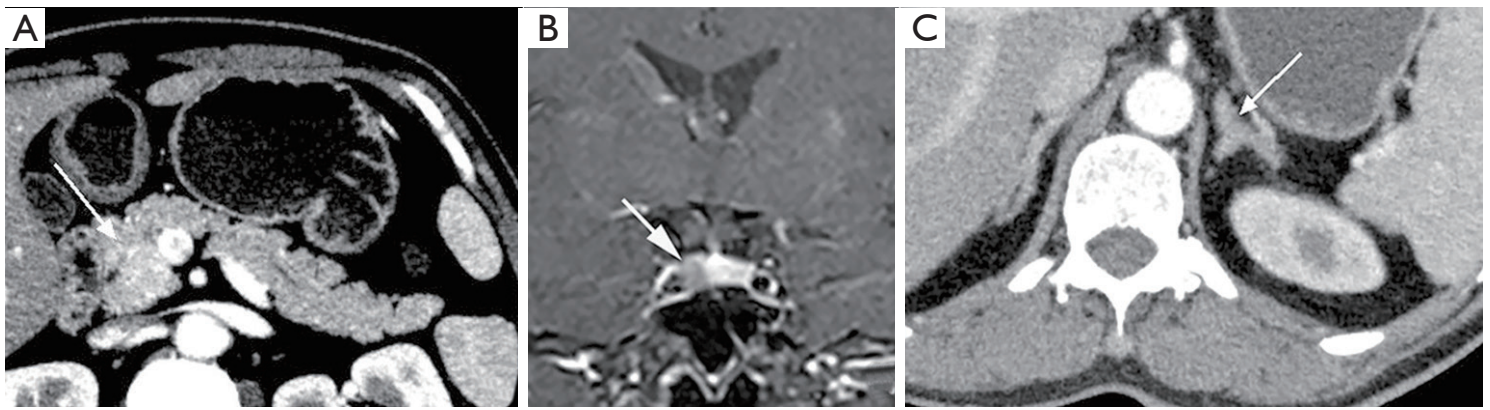

Figure 3 A 36-year-old woman presented with dizziness and was later diagnosed with MEN1. (A) Axial contrast-enhanced CT image showed an obviously enhanced nodule (arrow) in the pancreatic head. The lesion was later pathologically confirmed as insulinoma. (B) Coronal contrast-enhanced T1WI image showed a mildly enhanced nodule (arrow) on the right side of the pituitary. The lesion was clinically diagnosed as microadenoma. (C) Axial contrast-enhanced CT image showed left adrenal gland thickening (arrow), which was pathologically confirmed as adrenal hyperplasia. MEN1, multiple endocrine neoplasia type 1; CT, computed tomography; T1WI, T1 weighted imaging. 

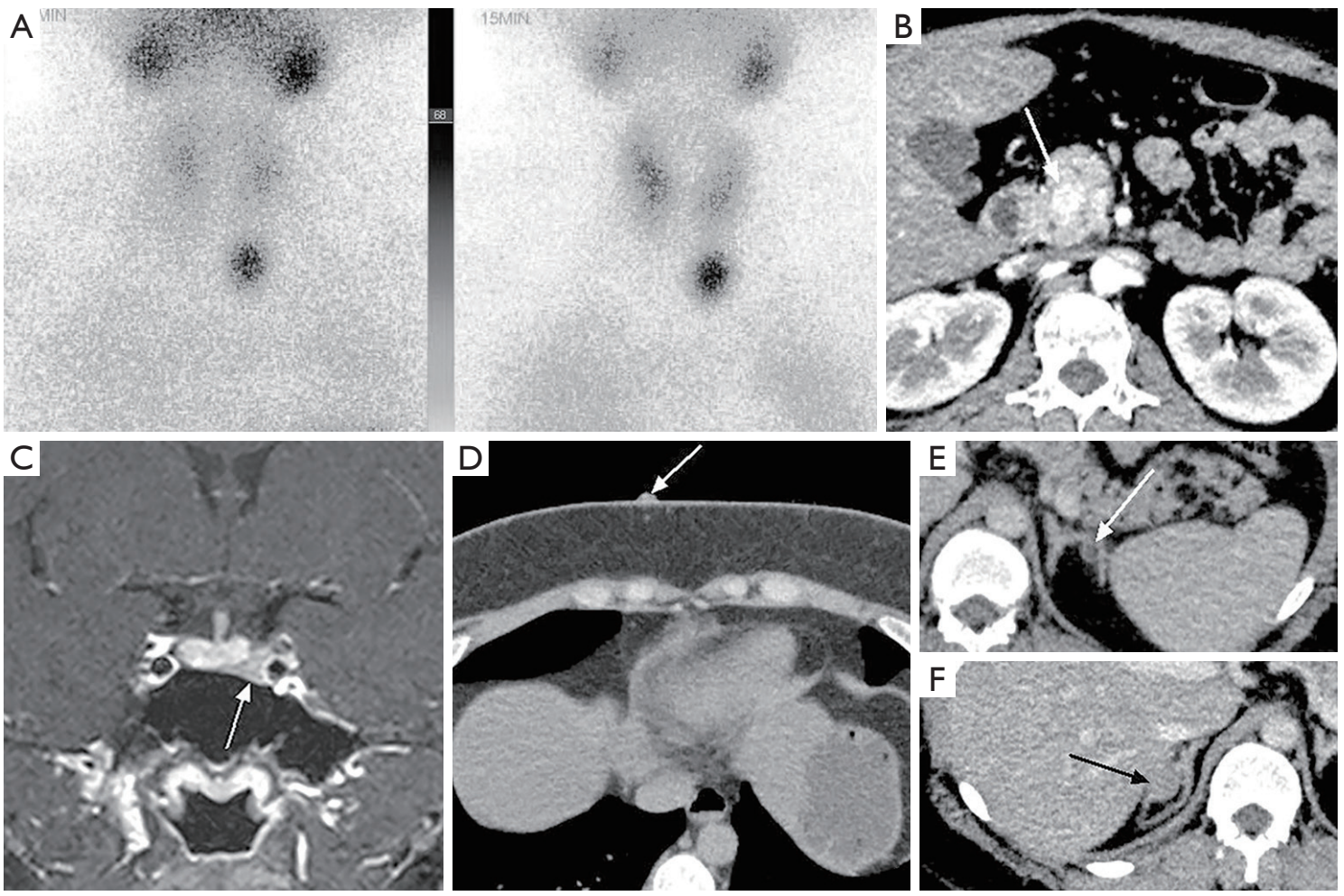

Figure 4 A 25-year-old female patient presented with galactorrhea, menstrual disorders, and obesity. She was later diagnosed with MEN1. (A) 99m Tc-MIBI image of the parathyroid glands was positive. (B) Axial contrast-enhanced CT image showed an obviously enhanced nodule (arrow) in the pancreatic head. (C) Coronal contrast-enhanced $\mathrm{T}_{1} \mathrm{WI}$ imaging showed a low-enhanced nodule (arrow) in the lowerleft part of the anterior pituitary. Clinically, it was diagnosed as pituitary microadenoma. (D) Axial CT image showed a multiple isodensity small nodule (arrow) in the abdominal wall, which was pathologically shown to be dermatofibroma. (E,F) Axial contrast-enhanced CT image showed mildly enhanced nodules (arrows) on both sides of the adrenal glands. MEN1, multiple endocrine neoplasia type 1; CT, computed tomography; $T_{1}$ WI, T1 weighted imaging.

and recurrent. Insulinomas are mostly benign, occurring in $10-15 \%$ of MEN1 patients, and the age of onset is younger than 40 years (especially younger than 20 years) $(1,24,25)$. The typical symptom of insulinoma is Whipple's triad, including hypoglycemia, low plasma glucose levels at onset, and disappearance of symptoms after glucose supplementation. Recurrent hypoglycemia attacks may be the initial manifestation of MEN1 (26), and these attacks may induce seizures. Insulinomas should be considered in patients with endocrine disorders complicated with refractory epilepsy (27). There are generally no clinical symptoms or biochemical changes for nonfunctioning tumors, and the tumors are usually detected in imaging examinations.

Gastro-entero-pancreatic endocrine tumors suspected to be MEN1-related should be tested according to the following biochemical screening program: serum gastrin, fasting blood glucose, insulin, C-peptide, glucagon, vascular intestinal polypeptide, pancreatic polypeptide, and chromogranin A. In terms of imaging, CT, magnetic resonance imaging (MRI), endoscopic ultrasonography (EUS), and somatostatin receptor scintigraphy are usually used. Lesions may sometimes be detected by gastroscopy. Multifocal lesions can be observed on CT images, mostly presenting as small, circular masses with clear boundaries on the pancreas and/or duodenum. Small masses are usually homogeneous, while larger masses may be heterogeneous due to cystic degeneration, necrosis, and calcification. Contrast-enhanced CT imaging showed that the enhancement of the masses was significantly higher than that of the surrounding normal tissue in the arterial phase (a few tumors had poor blood supply) (Figures 1B, C,2C,D,3A,4B) (5).

Gastric neuroendocrine neoplasms occurring in MEN1 
patients are related to hypergastrinemia associated with Zollinger-Ellison syndrome and complicated genetic changes, which are called gastric neuroendocrine neoplasms type II. The tumors originate from the proliferation of enterochromaffin-like (ECL) cells in the gastric fundus and body, and the lesions are located in the mucosa and submucosa. Gastric neuroendocrine neoplasms type II are usually multifocal and small $(1-2 \mathrm{~cm})$ and are often found and diagnosed via gastroscopy (Figure 1E). The lesions can also be detected through CT and MRI (Figure 1D). When multiple polypoid changes are found in the stomachs of MEN1 patients, attention should be given to discerning whether patients are complicated with gastric neuroendocrine neoplasm type II $(28,29)$.

\section{Pituitary tumors}

Pituitary tumors associated with MEN1 occur in 10-60\% of MEN1 patients and are generally found in the anterior pituitary (7). The incidence of pituitary tumors associated with MEN1 is higher in women than it is in men (1). The clinical manifestations of pituitary tumors are related to the hormones secreted by the tumor and the size of the tumor. Among pituitary tumors associated with MEN1, $60 \%$ secrete prolactin, about $25 \%$ secrete growth hormone, and $5 \%$ secrete corticotropin (30). Therefore, the clinical symptoms can manifest as hyperprolactinemia (amenorrhea, lactation, infertility), acromegaly, or Cushing's disease (1). Also, tumors may compress adjacent structures, which can cause visual impairment or hypopituitarism.

In terms of laboratory tests, the focus is the levels of prolactin and insulin-like growth factor. In terms of imaging, pituitary MRI is usually implemented to observe pituitary lesions. For microadenomas, the lesions sometimes cannot be directly observed; rather, their existence in the pituitary is indicated by swelling of the superior margin of the pituitary or collapse of the sellar floor. Conversely, macroadenoma can be directly observed, and the tumor may be gourd-shaped due to the growth of the suprasellar. On $T_{1}$ weighted imaging $\left(T_{1} W I\right)$, the adenoma generally shows low signal intensity, and on $\mathrm{T}_{2} \mathrm{WI}$, it has equal or high signal intensity. Contrast-enhanced images show that the adenoma is enhanced, and the signal intensity is lower than that of the normal pituitary (Figures $1 F, 2 B, 3 B, 4 C)(5)$.

\section{Other tumors associated with MEN1}

Patients with MEN1 often develop cutaneous tumors. Finding cutaneous tumors in suspicious patients can facilitate early diagnosis. Facial angiofibromas occur in
$22-88 \%$ of MEN1 patients, collagenomas occur in $0-72 \%$ of MEN1 patients, and lipomas occur in approximately $33 \%$ of MEN1 patients (1). Patients with MEN1 may also present with dermatofibroma (Figure $4 E$ ). Other tumors, such as adrenal cortex masses (Figures 1G,3C,4E,F), carcinoid tumors, and meningiomas are often found in imaging examinations without obvious corresponding clinical symptoms (1).

\section{MEN2}

The MEN2 syndrome includes MEN2A, MEN2B, and FMTC, and the prevalence is $2-5$ per 100,000 people (12). The main feature of MEN2 is MTC, which occurs in all 3 subtypes with high penetrance. The age of onset of MTC related to MEN2 is younger than that of sporadic MTC cases (31). The aggressiveness of MTC varies across different subtypes, and the order of aggressiveness from high to low is MEN2B, MEN2A, and FMTC (7). Caused by the abnormal proliferation of parathyroid gland C cells, MTC secrete too much calcitonin, often multicentric and bilateral (13).

\section{MEN2A}

Also called Sipple syndrome, MEN2A has a prevalence of 1 per 25,000 people (9). The main manifestations of MEN2A are MTC and pheochromocytoma (Figures 5,6). More than $90 \%$ of MEN2A patients develop MTC, and 50\% develop pheochromocytoma (7). There are some clinical variants of MEN2A, including MEN2A with cutaneous lichen amyloidosis and MEN2A with Hirschsprung disease (32).

\section{MTC}

The clinical manifestations of MTC are mainly diarrhea, flushing caused by excessive secretion of calcitonin, neck masses, and poor breathing caused by neck masses. Biochemical examination often suggests elevated calcitonin and abnormally elevated carcinoembryonic antigen. The thyroid is generally examined initially by neck ultrasound (12), the images of which display hypoechoic nodules with calcification. The positional relationship between the tumor and the surrounding tissues can be observed on CT images. The CT images also show irregular and inhomogeneous low-density nodules with unclear boundaries and calcification. Contrast-enhanced CT images show inhomogeneous and obvious enhancement of the mass (Figures $5 A, B, 6 A, C$ ). Whole-body radionuclide imaging can provide a basis for tumor staging (5). 

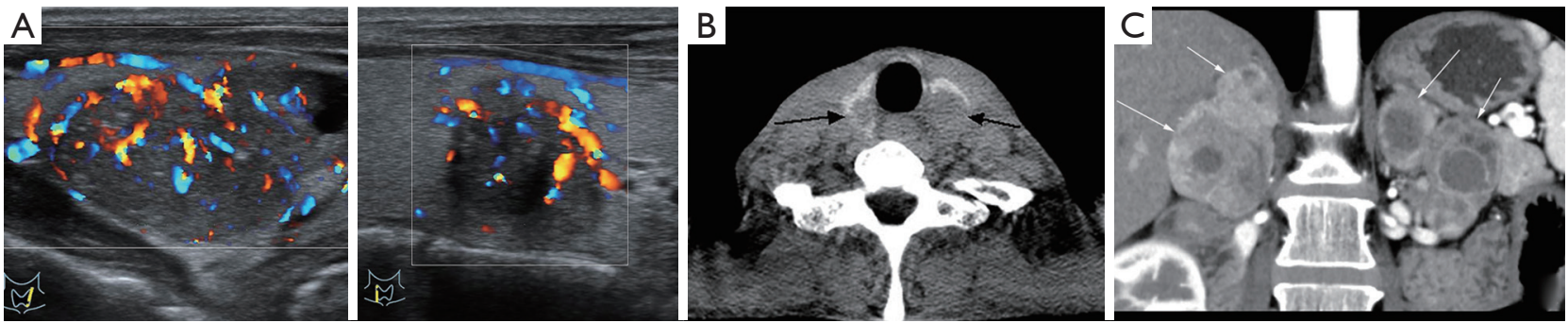

Figure 5 A 45-year-old man presented with repeated constipation and dizziness. He was later diagnosed with MEN2A. (A) Hypoechoic nodules with calcification could be seen on both sides of the thyroid gland on the ultrasound image. (B) Axial CT image showed lowdensity nodules (black arrows) on both sides of the thyroid gland. They were pathologically confirmed as medullary thyroid carcinoma. (C) Contrast-enhanced coronal reconstruction image showed the heterogeneous enhancement masses (arrows) in both adrenal glands. The lesions were pathologically confirmed as adrenal pheochromocytoma. MEN2A, multiple endocrine neoplasia type 2A; CT, computed tomography.
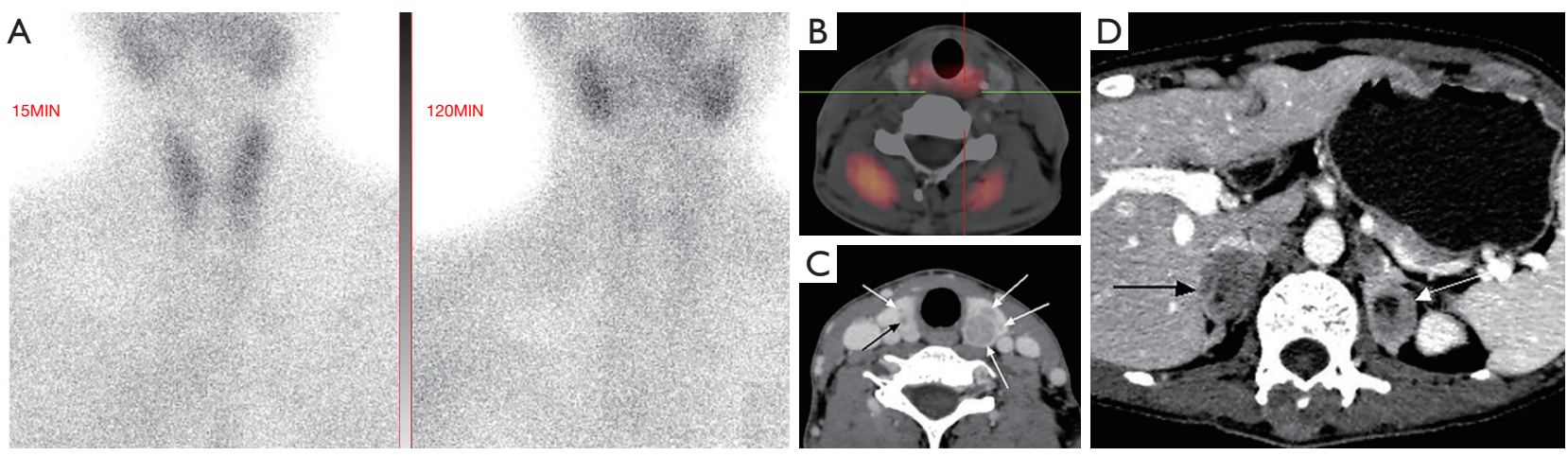

Figure 6 A 45-year-old man presented with emaciation and loss of appetite. He was later diagnosed with MEN2A. (A) 99mTc-MIBI image showed bilateral thyroid enlargement, and some nodules were "cool nodules". Also, the lesions were pathologically confirmed as medullary thyroid carcinoma. (B) CT and fusion images showed that image agents were concentrated in the parathyroid area. Clinically, the lesion was diagnosed as parathyroid hyperplasia. (C) Axial contrast-enhanced CT image showed that several low-density nodules (arrows) in the bilateral thyroid gland were uneven and obviously enhanced. The enhancement degree was lower than that of normal thyroid tissue. (D) Axial contrast-enhanced CT image showed inhomogeneous enhancement of multiple nodules (arrows) on both sides of the adrenal gland. The lesions were pathologically confirmed as bilateral adrenal pheochromocytomas. MEN2A, multiple endocrine neoplasia type 2A; CT, computed tomography

\section{Pheochromocytoma}

Pheochromocytomas related to MEN2A are mostly benign and rarely metastatic, usually occurring in bilateral adrenal glands $(6,12)$. The clinical manifestations include palpitations, elevated blood pressure, headache, and sweating. Biochemical examination shows abnormally elevated concentrations of catecholamines and their metabolites in plasma. Adrenal gland lesions are usually investigated by CT scan. Pheochromocytoma is characterized by a uniform density of small tumors, similar to the density of the kidney. Large tumors may have uneven density due to hemorrhage, necrosis, calcification, and cystic degeneration. In the contrast-enhanced CT examination, the tumor is significantly enhanced (Figures 5C,6D) (5).

\section{Other diseases associated with MEN2A}

HPT occurs in 20-30\% of MEN2A patients, generally without obvious symptoms, and is found by biochemical and image examination (Figure 6B) (7). The main cutaneous disease involved in MEN2A is lichen amyloidosis, which often occurs simultaneously as pheochromocytoma. The clinical manifestations are itchy and scaly rash on the upper 

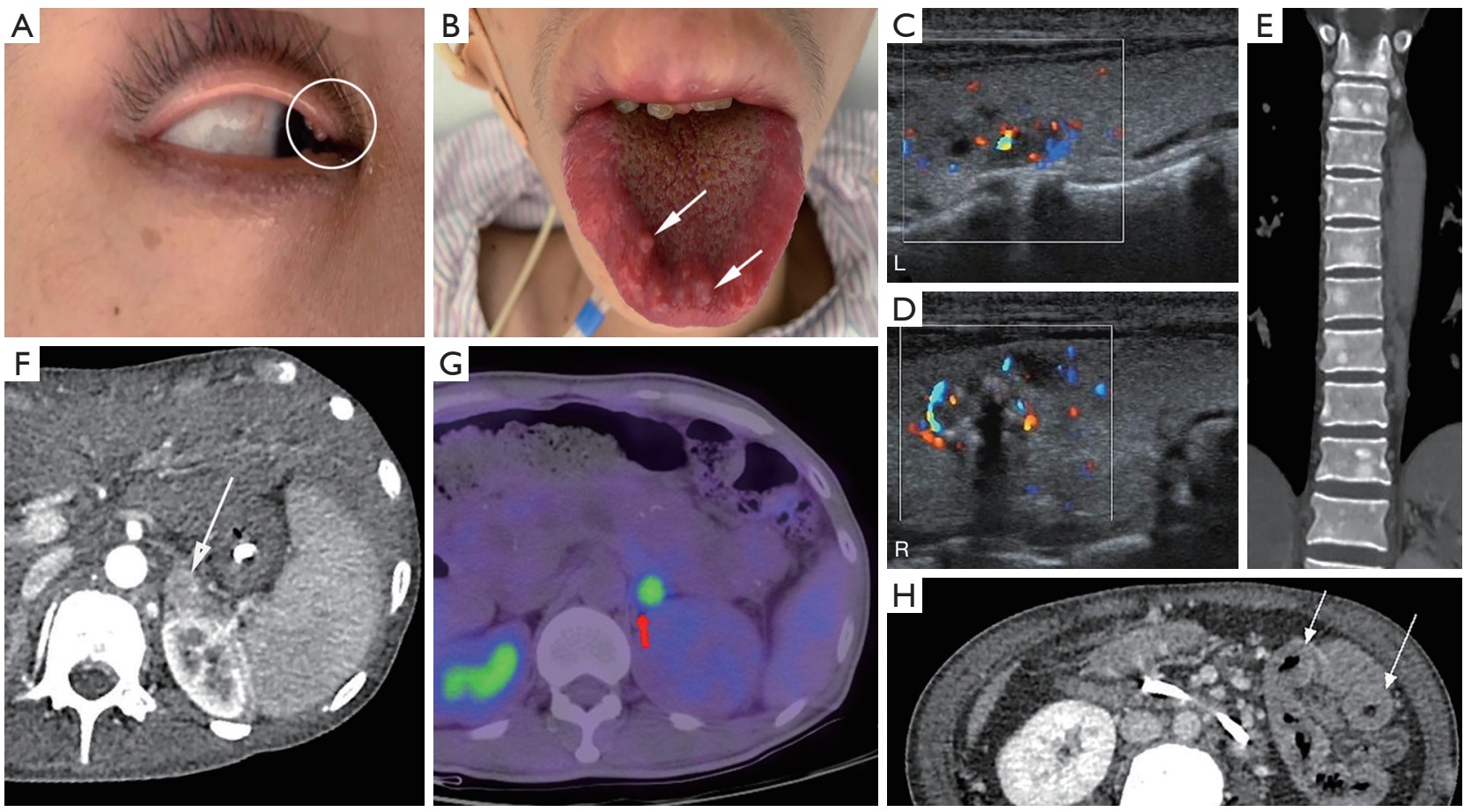

Figure 7 A 23-year-old male patient presented with abdominal pain and was later diagnosed with MEN2B. (A,B) Inspection showed multiple eyelid masses (circle) and tongue mucosal tumor-like hyperplasia (arrows). The lesions were pathologically confirmed as neuromas of the eyelids and mucosal neuromas of the tongue. (C,D) Ultrasound images showed thyroid lesions with mixed echoes, calcification, unclear boundaries, and abundant blood supply. (E) Coronal reconstruction image showed multiple high-density nodules on the thoracic vertebrae. They were pathologically confirmed as bone metastases of MTC. (F) Contrast-enhanced CT image showed a significantly enhanced roundlike nodule (arrow) in the left adrenal junction, which was pathologically confirmed as adrenal pheochromocytomas. (G) The nodule (arrow) in the left adrenal junction was positive on a somatostatin receptor image. $(\mathrm{H})$ Axial contrast-enhanced CT image showed that the small intestine wall was thickened and uniformly strengthened in segments (arrows). The lesions were pathologically confirmed as multiple submucosal gangliomas of the intestine. The RET gene mutation was detected in the genetic examination. MEN2B, multiple endocrine neoplasia type 2B; MTC, medullary thyroid carcinoma; CT, computed tomography; RET, rearranged during transfection proto-oncogene.

back (4). It has also been reported that MEN2A-related cutaneous diseases include dermal hyperplasia and multiple sclerosing fibromas (33). Approximately 7\% of MEN2A patients develop Hirschsprung disease, which is caused by abnormal development of intestinal ganglion cells and manifested as constipation (it should be distinguished from gastrointestinal neuromas related to MEN2B patients) (32).

\section{MEN2B}

Also known as Wagenmann-Froboese syndrome, MEN2B involves MTC and pheochromocytoma. The following distinguishing points from MEN2A: (I) MTC related to MEN2B are more aggressive and penetrative. Almost 100\% of MEN2B patients will develop MTC. (II) MEN2B patients generally do not develop the parathyroid disease. (III) MEN2B patients have some special manifestations that always occur before MTC and pheochromocytoma, and timely detection is helpful for the early diagnosis of MEN2B $(4,34,35)$. They include "Marfanoid" body habitus, gastrointestinal ganglion neuroma, multiple mucosal neuromas (lips, tongue, eyelids, conjunctiva), protruding lips, corneal nerve thickening, and bone abnormalities (such as joint laxity) (Figure 7) (4). It is worth noting that abdominal discomfort, such as constipation, in patients with MEN2B may be caused by gastrointestinal ganglioneuroma and Hirschsprung disease (36).

\section{FMTC}

As FMTC only involves the thyroid gland, it can also 

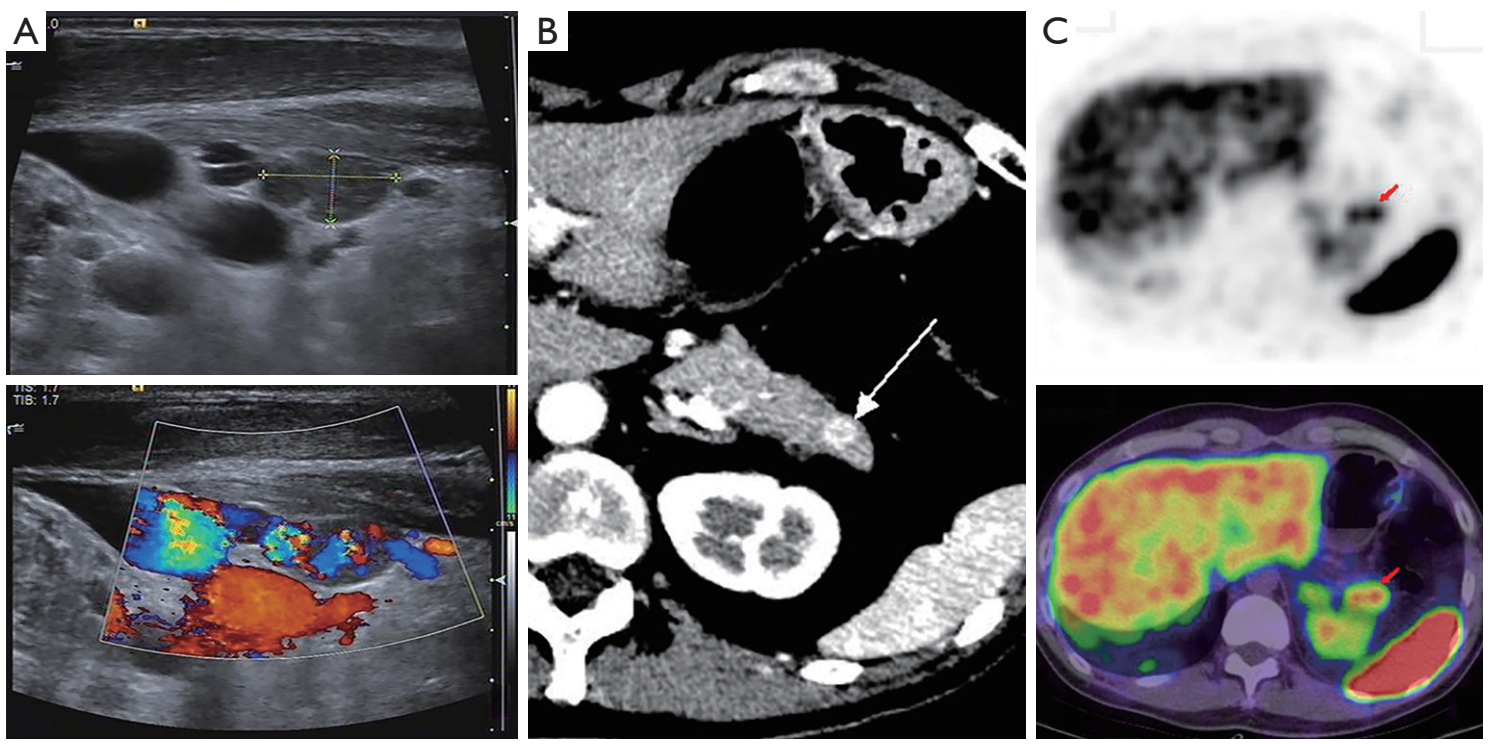

Figure 8 A 35-year-old male with MEN4 presented with diarrhea and atypical thymic carcinoid (resected). He was later diagnosed with MEN4. (A) Ultrasound image showed that there was a hypoechoic nodule near the lower pole of the right thyroid lobe with abundant blood supply. The nodule was pathologically confirmed as parathyroid adenoma. (B) Axial contrast-enhanced CT image showed an obviously enhanced nodule (arrow) in the tail of the pancreas. (C) On the somatostatin receptor image, the lesion (arrows) in the tail of the pancreas was positive. The lesion was pathologically confirmed as a neuroendocrine tumor. Genetic testing indicated harmless mutations in MEN1 that did not cause amino acid changes. Later, genetic testing was performed to indicate pathogenic mutations of CDKN1B. MEN4, multiple endocrine neoplasia type 4; CT, computed tomography; CDKN1B, cyclin-dependent kinase (CDK) inhibitor $1 \mathrm{~b}$ gene.

be considered a mild variant of MEN2A. To avoid misdiagnosis, FMTC should strictly follow the diagnostic criteria. Some FMTC patients are also complicated with Hirschsprung disease (37).

\section{MEN4}

The clinical manifestations of MEN4 are similar to those of MEN1. HPT and pituitary tumors are common manifestations of MEN4. Other tumors, such as gastroentero-pancreatic endocrine tumors, adrenal tumors, lipomas, meningiomas, and carcinoids, can also be found in patients with MEN4. According to the reported cases, some phenotypic characteristics of MEN4 have been summarized. Firstly, the onset of tumors related to MEN4 is later than those of MEN1. HPT usually occurs at over 40 years old (the earliest age of onset in reported cases is 15 years old). Secondly, the incidence of gastro-entero-pancreatic endocrine tumors related to MEN4 is lower than that of those related to MEN1. Additionally, prolactin tumors in pituitary tumors were less common in MEN4 than MEN1 (Figure 8) (16,17).

\section{Diagnosis}

\section{MEN1}

Patients who meet any of the following 3 diagnostic criteria can be diagnosed with MEN1. (I) Clinical diagnosis: the patient has 2 or more of the 3 main tumors (parathyroid tumor, pituitary tumor, gastro-entero-pancreatic endocrine tumor). (II) Family diagnosis: the patient meets the clinical diagnostic criteria and has a first-degree relative (including parents, children, and siblings) who has 1 of the 3 main tumors. (III) Genetic diagnosis: genetic testing of the patient reveals a mutation in the MEN1 gene (1).

\section{MEN2}

It is necessary to carry out genetic testing for patients suspected of having MEN2. Mutations of RET are the basis for the genetic diagnosis of MEN2, and classification should be made according to the genotype-phenotype relationship. It is possible to diagnose MEN2 clinically, and the following are the clinical diagnostic criteria of the three subtypes of MEN2 (37,38): 
* MEN2A: patients with 2 or more major tumors (MTC, pheochromocytoma, parathyroid tumor) can be clinically diagnosed with MEN2A, and attention should be paid to excluding MEN2B diagnosis in advance (37).

* MEN2B: clinically, MEN2B is usually diagnosed by identifying MTC, pheochromocytoma, and some special manifestations of MEN2B ("Marfanoid" body habitus, multiple mucosal neuromas, corneal nerve thickening) (37).

* FMTC: the patient only has 1 endocrine gland tumor, namely, MTC, and there are 4 or more cases of MTC without MEN1, MEN2A, or MEN2B signs in the same family (37).

\section{MEN4}

Patients with the clinical MEN1 phenotype who do not have MEN1 mutations in the genetic detection but have CDKN1B mutations can be diagnosed as MEN4 (17).

Patients clinically diagnosed as MEN, their bloodrelated family members, and patients suspected of MEN should undergo genetic testing. Genetic testing can be used for early diagnosis, identification of asymptomatic carriers, and preimplantation diagnosis. There have been successful cases of preimplantation genetic diagnosis $(11,39,40)$.

\section{Discussion}

Patients with hereditary MEN syndromes should be differentiated from sporadic cases. Particular features of suspicious cases should arouse the vigilance of clinicians.

(I) MEN1: Parathyroid hyperplasia or adenoma associated with MEN1 occurs earlier than in sporadic cases and often occurs in multiple glands. The onset age of gastro-entero-pancreatic neuroendocrine tumors related to MEN1 is earlier than that of sporadic cases. Gastro-entero-pancreatic neuroendocrine tumors usually manifest as multifocal and small lesions. Therefore, MEN1 should be considered in any individual with the following features: HPT at less than 40 years old, polyglandular or recurrent HPT, gastro-entero-pancreatic neuroendocrine tumors at less than 40 years old, multifocal gastro-entero-pancreatic neuroendocrine tumors, gastrinoma, gastro-entero-pancreatic neuroendocrine tumors with nephrolithiasis or hypercalcemia. Also, patients with endocrine diseases or endocrine family history with skin masses should also be mindful of MEN1.

(II) MEN2: 25\% of MTC patients are associated with MEN2, so all MTC and C cell hyperplasia patients should be tested for the RET gene. Bilateral adrenal pheochromocytoma is often associated with familial hereditary diseases and requires clinical vigilance. Lichen amyloidosis on the back skin is a characteristic manifestation of MEN2A. If detected in physical examination, clinicians should be alert to the possibility of MEN2A. The special manifestations (Marfanoid habitus, mucosal neuromas, protruding lips, gastrointestinal ganglion neuroma) of MEN2B provide an important clue for the early diagnosis of MEN2B.

For patients with typical phenotypes of MEN2 but no RET gene mutation, we can make the clinical diagnosis of MEN2 when they meet the clinical diagnostic criteria of MEN2, which we have described in the diagnosis section of this article. In the instance of such patients, their highrisk relatives should be regularly screened. Screening for MTC includes neck ultrasound, basal and/or irritant calcitonin determination. Pheochromocytoma is screened by measuring plasma or 24-hour urine concentrations of catecholamines and their metabolites. Also, periodic tumor tests should be performed in patients who are suspected of MEN2 but do not meet clinical or genetic diagnostic criteria.

\section{Conclusions}

The MEN syndromes affect the patient's quality of life and can lead to chronic inflammation and immunosuppression $(41,42)$. Clinicians should understand the characteristics of the disease to make a timely and correct diagnosis. According to the characteristics of each type of MEN, some memorization methods can be summarized (Figure 9). The MEN1 type mainly involves parathyroid tumors, gastro-entero-pancreatic endocrine tumors, and pituitary tumors, which can be remembered as " $2 \mathrm{P} 1 \mathrm{G}$ ". The clinical phenotype of MEN4 is similar to that of MEN1, but the genes involved are different. Thus far, there have been few studies on MEN4. The MEN2A type mainly involves MTC, pheochromocytoma, and parathyroid tumors, which can be simply remembered as "2P1M". Meanwhile, MEN2B mainly manifests as MTC, pheochromocytoma, Marfanoid habitus, and mucosal neuroma, which can be remembered as " $1 \mathrm{P} 3 \mathrm{M}$ ". When a patient is suspected of 


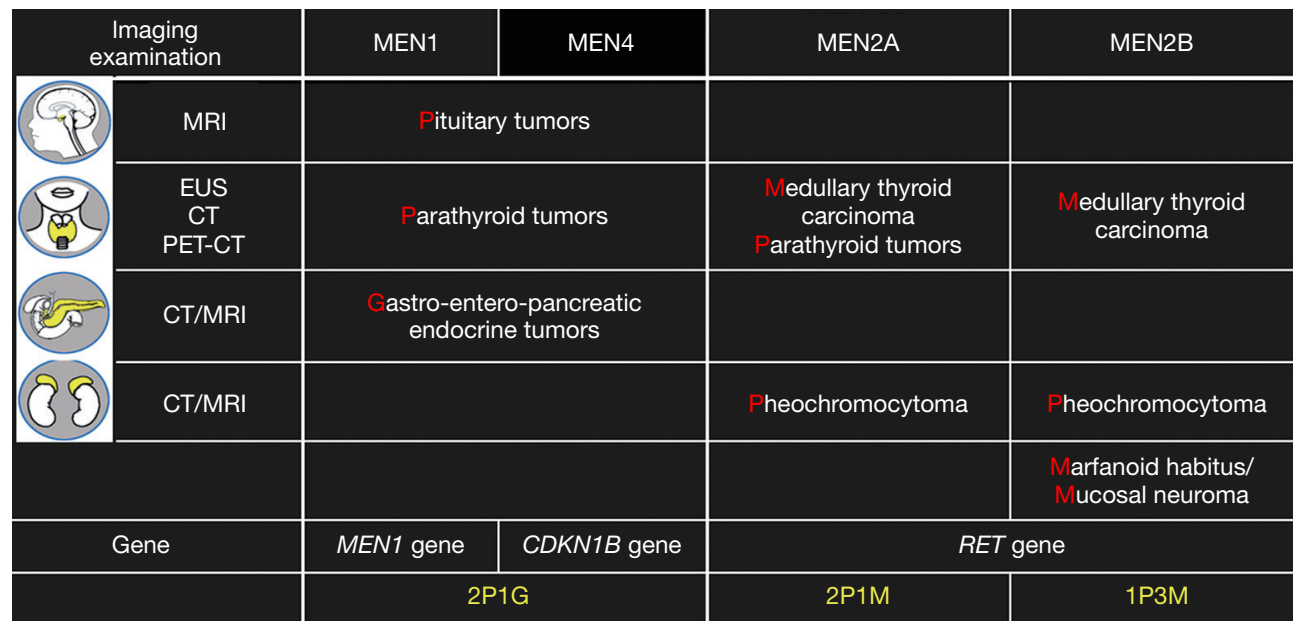

Figure 9 Characteristics of each type of MEN syndromes. MEN, multiple endocrine neoplasia; MRI, magnetic resonance imaging; CT, computed tomography; EUS, endoscopic ultrasonography; PET, positron emission tomography.

having MEN syndromes, comprehensive biochemical and imaging examinations should be performed according to the relevant disease spectrum to detect the underlying disease as soon as possible.

\section{Acknowledgments}

We would like to acknowledge J. Jones and J. Chapnick for checking and editing the manuscript.

Funding: This work was supported by the National Natural Science Foundation of China (81801761 to YL).

\section{Footnote}

Reporting Checklist: The authors have completed the Narrative Review reporting checklist. Available at http:// dx.doi.org/10.21037/atm-21-1165

Peer Review File: Available at http://dx.doi.org/10.21037/ atm-21-1165

Conflicts of Interest: All authors have completed the ICMJE uniform disclosure form (available at http://dx.doi. org/10.21037/atm-21-1165). The authors have no conflicts of interest to declare.

Ethical Statement: The authors are accountable for all aspects of the work in ensuring that questions related to the accuracy or integrity of any part of the work are appropriately investigated and resolved.
Open Access Statement: This is an Open Access article distributed in accordance with the Creative Commons Attribution-NonCommercial-NoDerivs 4.0 International License (CC BY-NC-ND 4.0), which permits the noncommercial replication and distribution of the article with the strict proviso that no changes or edits are made and the original work is properly cited (including links to both the formal publication through the relevant DOI and the license). See: https://creativecommons.org/licenses/by-nc-nd/4.0/.

\section{References}

1. Thakker RV, Newey PJ, Walls GV, et al. Clinical practice guidelines for multiple endocrine neoplasia type 1 (MEN1). J Clin Endocrinol Metab 2012;97:2990-3011.

2. Margraf RL, Crockett DK, Krautscheid PM, et al. Multiple endocrine neoplasia type 2 RET protooncogene database: repository of MEN2-associated RET sequence variation and reference for genotype/phenotype correlations. Hum Mutat 2009;30:548-56.

3. Pellegata NS, Quintanilla-Martinez L, Siggelkow H, et al. Germ-line mutations in p27Kip1 cause a multiple endocrine neoplasia syndrome in rats and humans. Proc Natl Acad Sci U S A 2006;103:15558-63.

4. Clark P. Multiple Endocrine Neoplasia Syndromes. J Infus Nurs 2015;38 Suppl 6:S36-42.

5. Grajo JR, Paspulati RM, Sahani DV, et al. Multiple Endocrine Neoplasia Syndromes: A Comprehensive Imaging Review. Radiol Clin North Am 2016;54:441-51.

6. McDonnell JE, Gild ML, Clifton-Bligh RJ, et al. Multiple 
endocrine neoplasia: an update. Intern Med J 2019;49:954-61.

7. Brandi ML, Gagel RF, Angeli A, et al. Guidelines for diagnosis and therapy of MEN type 1 and type 2. J Clin Endocrinol Metab 2001;86:5658-71.

8. Lemos MC, Thakker RV. Multiple endocrine neoplasia type 1 (MEN1): analysis of 1336 mutations reported in the first decade following identification of the gene. Hum Mutat 2008;29:22-32.

9. Romei C, Pardi E, Cetani F, et al. Genetic and clinical features of multiple endocrine neoplasia types 1 and 2.J Oncol 2012;2012:705036.

10. De Paoli-Iseppi R, Prentice L, Marthick JR, et al. Multiple endocrine neoplasia type 1: clinical correlates of MEN1 gene methylation. Pathology 2018;50:622-8.

11. Marini F, Giusti F, Brandi ML. Multiple endocrine neoplasia type 1: extensive analysis of a large database of Florentine patients. Orphanet J Rare Dis 2018;13:205.

12. Täieb D, Kebebew E, Castinetti F, et al. Diagnosis and preoperative imaging of multiple endocrine neoplasia type 2: current status and future directions. Clin Endocrinol (Oxf) 2014;81:317-28.

13. Walls GV. Multiple endocrine neoplasia (MEN) syndromes. Semin Pediatr Surg 2014;23:96-101.

14. Zhang XW, Wang JY, Zhang YB, et al. Genotypephenotype correlations in multiple endocrine neoplasia type 2. Zhonghua Er Bi Yan Hou Tou Jing Wai Ke Za Zhi 2016;51:538-41.

15. Ying R, Feng J. Clinical significance of RET mutation screening in a pedigree of multiple endocrine neoplasia type 2A. Mol Med Rep 2016;14:1413-7.

16. Chevalier B, Odou MF, Demonchy J, et al. Multiple Endocrine Neoplasia Type 4: Novel CDNK1B variant and immune anomalies. Ann Endocrinol (Paris) 2020;81:124-5.

17. Frederiksen A, Rossing M, Hermann P, et al. Clinical Features of Multiple Endocrine Neoplasia Type 4: Novel Pathogenic Variant and Review of Published Cases. J Clin Endocrinol Metab 2019;104:3637-46.

18. Jhawar S, Lakhotia R, Suzuki M, et al. Clinical presentation and management of primary ovarian neuroendocrine tumor in multiple endocrine neoplasia type 1. Endocrinol Diabetes Metab Case Rep 2019;2019:19-0040.

19. Misgar RA, Sahu D, Purra S, et al. Multiple Uterine Leiomyomas in Multiple Endocrine Neoplasia Type 1 with a Novel MEN1 Gene Mutation. J Hum Reprod Sci 2020;13:75-7.

20. Lou L, Zhou L, Wang W, et al. Atypical ovarian carcinoid tumor with widespread skeletal metastases: a case report of multiple endocrine neoplasia type 1 in a young woman. BMC Cancer 2019;19:1107.

21. Manoharan J, Bollmann C, Kann PH, et al. Gender Differences in Multiple Endocrine Neoplasia Type 1: Implications for Screening? Visc Med 2020;36:3-9.

22. Norton JA, Krampitz G, Jensen RT. Multiple Endocrine Neoplasia: Genetics and Clinical Management. Surg Oncol Clin N Am 2015;24:795-832.

23. Slouma M, Abbes M, Dhahri R, et al. Multiple endocrine neoplasia type 1 revealed by a hip pathologic fracture. Clin Rheumatol 2021;40:775-82.

24. Varas Lorenzo M, Cugat Andorra E, Capdevila Castillón J. Pancreatic neuroendocrine tumors. Rev Esp Enferm Dig 2017;109:480-1.

25. van Beek DJ, Nell S, Verkooijen HM, et al. Surgery for multiple endocrine neoplasia type 1-related insulinoma: long-term outcomes in a large international cohort. Br J Surg 2020;107:1489-99.

26. Okubo Y, Tono T, Fukamizu M, et al. Multiple Endocrine Neoplasia Type 1(MEN1)Presenting with Hypoglycemic Attacks - A Case Report. Gan To Kagaku Ryoho 2016;43:2196-8.

27. Kumawat BL, Sharma C, Shah MJ, et al. Multiple endocrine neoplasia type 1 presenting with refractory seizures. BMJ Case Rep 2017;2017:bcr2016218982.

28. Grozinsky-Glasberg S, Alexandraki KI, Angelousi A, et al. Gastric Carcinoids. Endocrinol Metab Clin North Am 2018;47:645-60.

29. Delle Fave G, Capurso G, Annibale B, et al. Gastric neuroendocrine tumors. Neuroendocrinology 2004;80 Suppl 1:16-9.

30. Trouillas J, Labat-Moleur F, Sturm N, et al. Pituitary tumors and hyperplasia in multiple endocrine neoplasia type 1 syndrome (MEN1): a case-control study in a series of 77 patients versus 2509 non-MEN1 patients. Am J Surg Pathol 2008;32:534-43.

31. Calender A; Groupe d'étude des Tumeurs Endocrines. Multiple endocrine neoplasia: genetic aspects. Bull Acad Natl Med 2010;194:81-95; discussion 95-6.

32. Wells SA Jr, Pacini F, Robinson BG, et al. Multiple endocrine neoplasia type 2 and familial medullary thyroid carcinoma: an update. J Clin Endocrinol Metab 2013;98:3149-64.

33. Alegría-Landa V, Jo-Velasco M, Robledo M, et al. Dermal Hyperneury and Multiple Sclerotic Fibromas in Multiple Endocrine Neoplasia Type 2A Syndrome. JAMA Dermatol 2017;153:1298-301. 
34. Redlich A, Lessel L, Petrou A, et al. Multiple endocrine neoplasia type 2B: Frequency of physical stigmata-Results of the GPOH-MET registry. Pediatr Blood Cancer 2020;67:e28056.

35. Nagaoka R, Sugitani I, Sanada M, et al. The Reality of Multiple Endocrine Neoplasia Type 2B Diagnosis: Awareness of Unique Physical Appearance Is Important. J Nippon Med Sch 2018;85:178-82.

36. Zhang L, Guo Y, Ye L, et al. Severe constipation as the first clinical manifestation in multiple endocrine neoplasia type $2 \mathrm{~B}$ : a case report and literature review. BMC Pediatr 2020;20:318.

37. Moline J, Eng C. Multiple endocrine neoplasia type 2: an overview. Genet Med 2011;13:755-64.

38. Krampitz GW, Norton JA. RET gene mutations (genotype and phenotype) of multiple endocrine neoplasia type 2 and familial medullary thyroid carcinoma. Cancer 2014;120:1920-31.

Cite this article as: Hu X, Guan J, Wang Y, Shi S, Song C, Li ZP, Feng ST, Chen J, Luo Y. A narrative review of multiple endocrine neoplasia syndromes: genetics, clinical features, imaging findings, and diagnosis. Ann Transl Med 2021;9(11):944. doi: 10.21037/atm-21-1165
39. Lima AD, Alves VR, Rocha AR, et al. Preimplantation genetic diagnosis for a patient with multiple endocrine neoplasia type 1: case report. JBRA Assist Reprod 2018;22:67-70.

40. Chen S, Li S, Zhang J, et al. Preimplantation Genetic Diagnosis of Multiple Endocrine Neoplasia Type 2A Using Informative Markers Identified by Targeted Sequencing. Thyroid 2018;28:281-7.

41. van Leeuwaarde RS, Pieterman CRC, May AM, et al. Health-Related Quality of Life in Patients with Multiple Endocrine Neoplasia Type 1. Neuroendocrinology 2021;111:288-96.

42. Grey J, Winter K. Patient quality of life and prognosis in multiple endocrine neoplasia type 2. Endocr Relat Cancer 2018;25:T69-77.

(English Language Editors: J. Jones and J. Chapnick) 